\title{
Sequencing and Characterization of Oligosaccharides Using Infrared Multiphoton Dissociation and Boronic Acid Derivatization in a Quadrupole Ion Trap
}

\author{
Michael Pikulski, Amanda Hargrove, Shagufta H. Shabbir, \\ Eric V. Anslyn, and Jennifer S. Brodbelt \\ Department of Chemistry and Biochemistry, The University of Texas at Austin, Austin, Texas, USA
}

\begin{abstract}
A simplified method for determining the sequence and branching of oligosaccharides using infrared multiphoton dissociation (IRMPD) in a quadrupole ion trap (QIT) is described. An IR-active boronic acid (IRABA) reagent is used to derivatize the oligosaccharides before IRMPD analysis. The IRABA ligand is designed to both enhance the efficiency of the derivatization reaction and to facilitate the photon absorption process. The resulting IRMPD spectra display oligosaccharide fragments that are formed from primarily one type of diagnostic cleavage, thus making sequencing straightforward. The presence of sequential fragment ions, a phenomenon of IRMPD, permit the comprehensive sequencing of the oligosaccharides studied in a single stage of activation. We demonstrate this approach for two series of oligosaccharides, the lacto-N-fucopentaoses (LNFPs) and the lacto-N-difucohexaoses (LNDFHs). (J Am Soc Mass Spectrom 2007, 18, 2094-2106) @ 2007 American Society for Mass Spectrometry
\end{abstract}

$\mathrm{O}$ ligosaccharides are in many ways more structurally complex than other biologically important molecules such as proteins and nucleic acids in that they are often highly branched and have several different linkage types between their fundamental monosaccharide units. Even excluding stereochemistry, a glycosidic bond between two hexoses can be formed at any of five positions. This results in a great number of structural possibilities that make characterization of oligosaccharides a challenging but vital task because they have important roles in numerous biological processes. Oligosaccharides are known to play roles in cell-cell signaling, cellular differentiation, regulation of biochemical pathways, viral replication, parasitic infection, immune response, and inflammation [1-7]. More than half of mammalian proteins are glycosylated [1], and the functions attributed to the oligosaccharides are broadly grouped as structural/modulatory and specific recognition by receptors [8]. For example, some oligosaccharides are involved in cell wall formation and have protective and stabilizing roles while others are very specific receptors for bacteria and viruses. In addition, the alteration of oligosaccharide expression has been associated with disease progression, suggesting that oligosaccharides may be used as biomarkers [9]. For instance, malignancy has been correlated with

Address reprint requests to Dr. J. S. Brodbelt, Department of Chemistry and Biochemistry, The University of Texas at Austin, 1 University Station A5300, Austin, TX 78712-0165, USA. E-mail: jbrodbelt@mail.utexas.edu changes in the glycosylation process, and some cell surface oligosaccharides are linked with prognosis in human cancers [10,11]. To fully understand the roles of glycosylation in these biological processes, it is critical to develop sensitive methods of characterization.

Mass spectrometry (MS) has become an invaluable tool for the structural determination of oligosaccharides mainly due to the minimal sample consumption and specificity of the information obtained. Matrix assisted laser desorption ionization (MALDI) time-of-flight (TOF) MS has been used for the analysis of native, metal adducted and derivatized oligosaccharides [12-15]. Structural isomers have been differentiated successfully by comparing the intensities of post-source decay (PSD) fragment ions. While MALDI-TOF-MS has provided some detailed information about isomers, more detailed structural analysis is generally performed by using tandem mass spectrometry.

Trapping instruments such as the quadrupole ion trap (QIT) and Fourier transform ion cyclotron resonance (FTICR) mass spectrometers have also been used for the analysis of oligosaccharides because of the ability to undertake $\mathrm{MS}^{\mathrm{n}}$ experiments, which is often required to fully map the branching patterns and sequences of oligosaccharides. Collisionally activated dissociation (CAD) of oligosaccharides primarily leads to fragment ions due to B-/Y- and C-/Z-type cleavages [15-27]. The number of cleavages in the first stage of activation is often limited, and thus frequently requires several stages of activation to obtain partial sequence 
information, particularly for larger oligosaccharides. In addition, $\mathrm{CAD}$ often leads to cleavages from both the reducing and nonreducing ends of oligosaccharides in addition to internal losses, thus complicating spectral interpretation. To provide more extensive structural information and alleviate the need for elaborate $\mathrm{MS}^{\mathrm{n}}$ strategies, other activation methods have also been applied to glycopeptides and oligosaccharides in trapping instruments. For example, Adamson et al. used electron capture dissociation (ECD) in an FTICR mass spectrometer to promote cross-ring over glycosidic cleavages and increase the amount of linkage information obtained [28]. Wolff et al. used electron detachment dissociation (EDD) to promote cross-ring cleavages and identify sites of sulfation in glycosaminooligosaccharide tetrasaccharides [29]. Most recently, Devakumar et al. used 157-nm UV light to photofragment glycans in an ion trap. Extensive high-energy cross-ring cleavages were observed that provided branching information [30].

Infrared multiphoton dissociation (IRMPD) is another alternative activation method that has generated substantial recent interest. Several studies have explored the use of IRMPD in an FTICR mass spectrometer for the analysis of oligosaccharides [16, 31-35]. For example, Lancaster et al. compared the IRMPD spectra of O-linked and N-linked type oligosaccharides and found that cross-ring cleavages were observed only for the N-type oligosaccharides that are not readily fragmented by using CAD [16]. Goldberg et al. used IRMPD in an FTICR instrument and developed a strategy using a computer algorithm to determine oligosaccharide sequence. While the spectra were informative, poor signal-to-noise ratios often made spectral interpretation difficult [31]. Fukui et al. used wavelength-tunable IRMPD in an FTICR mass spectrometer to determine the optimal dissociation wavelengths for sodium-cationized oligosaccharides [32]. While this technique is powerful, these types of lasers are not readily available. Zhang et al. used IRMPD in an FTICR instrument on oligosaccharide alditols and discovered specific fragments corresponding to structural motifs that revealed sequence and linkage information [33].

IRMPD has also been implemented in quadrupole ion traps for the analysis of flavonoids, antibiotics, peptides, and oligonucleotides [36-46] and affords a promising alternative to $\mathrm{CAD}$ for the characterization of oligosaccharides as described in the present study. The IRMPD process is independent of the rf trapping voltage, meaning that storage and detection of ions over a wide $\mathrm{m} / \mathrm{z}$ range is possible, including very low $\mathrm{m} / \mathrm{z}$ fragment ions at the same time as high $\mathrm{m} / \mathrm{z}$ precursors. Since IRMPD is not a collision-based method; there are fewer ion losses due to scattering. This reduction of ion losses is a critical feature when ion populations are low, which is often the case for oligosaccharides. Another potential advantage of IRMPD involves the ease of controlling photon flux to enhance or limit sequential fragmentation processes. Since IRMPD is not a resonant process, energy absorption by primary fragment ions during the activation period may result in secondary fragmentation, and the extent can be varied by adjusting the activation time or laser power. The secondary fragments often provide important structural information without the need for more elaborate $\mathrm{MS}^{\mathrm{n}}$ strategies. One drawback to IRMPD in a quadrupole ion trap stems from the relatively slow rate of energy accumulation (only $\sim 0.1 \mathrm{eV}$ per photon) that allows competition from collisional cooling due to the helium bath gas. In addition to other recent methods designed to overcome this drawback, such as thermally assisted IRMPD and collision-activated IRMPD [47-51], we have recently incorporated chromophores that absorb IR radiation very efficiently into analytes of interest. For example, we have explored the use of IR-active ligands (IRALs) for the enhancement of IRMPD efficiency of flavonoids in a quadrupole ion trap [36]. In this previous study, IR-active phosphonate groups were attached to chelating ligands that were then noncovalently bound to the analytes of interest via metal coordination in solution. The resulting complexes underwent highly efficient IRMPD. Companion FTIR and energy-variable CAD results confirmed that the chromogenic ligands were effective because of the high IR absorptivities of the phosphonate groups, thus providing strong IR chromophores for rapid energy accumulation. The use of a phosphonate-based IR chromophore is also exploited in the present study.

While it has become common practice to form chromophore-labeled carbohydrate derivatives for UV and fluorescence detection following chromatographic separation, various chemical derivatization strategies of oligosaccharides have also been used in conjunction with mass spectrometry to increase ionization sensitivity as well as yield more informative fragmentation patterns that reveal linkage and anomeric configuration [52-58]. Some of the derivatization techniques used for ESI-MS analysis of carbohydrates include permethylation for linkage information $[52,53]$, reductive amination for attachment of chromophores for HPLC detection [54], $\mathrm{p}$-aminobenzoic ethyl ester derivatization via formation of a glycosylamine for determination of linkage and branching information [55], and modification of cis-diol groups of disaccharides with boronic acids for stereochemical determination [56, 57]. Most recently, phenylboronic acid was used as a reagent ion for the analysis of biologically active diol compounds by desorption electrospray ionization (DESI) MS [58].

In the present study, we develop a method for the characterization of oligosaccharides in a quadrupole ion trap using IRMPD to attain complete sequencing and branching information. In contrast to other recent methods developed to promote cross-ring cleavages to enhance linkage information, the goal of our study was to simplify and limit the spectra to glycosidic cleavages for comprehensive and straightforward sequencing. The sequence and branching positions of two series of isomeric oligosaccharides were determined (Figure 1). 


\section{LNFP Glycans}

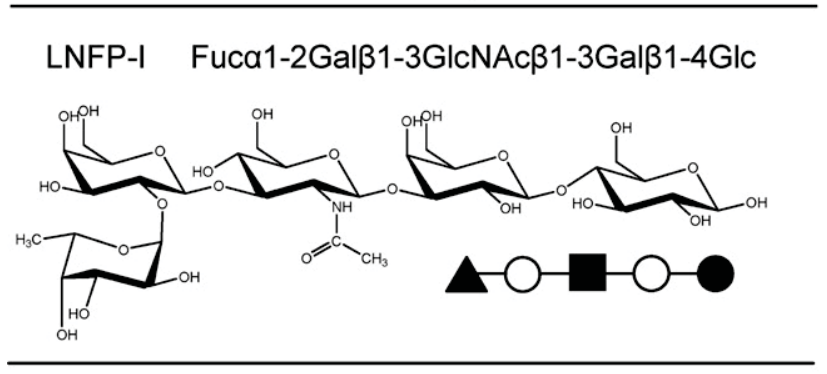

LNFP-II Galß1-3(Fuca1-4)GIcNAcß1-3Galß1-4Glc

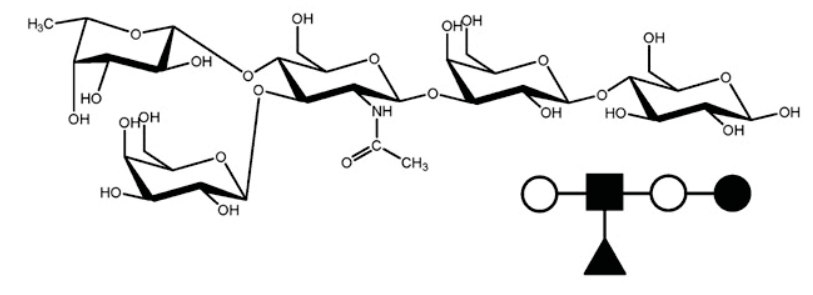

LNFP-III Galß1-3(Fuca1-3)GIcNAcß1-3Galß1-4Glc
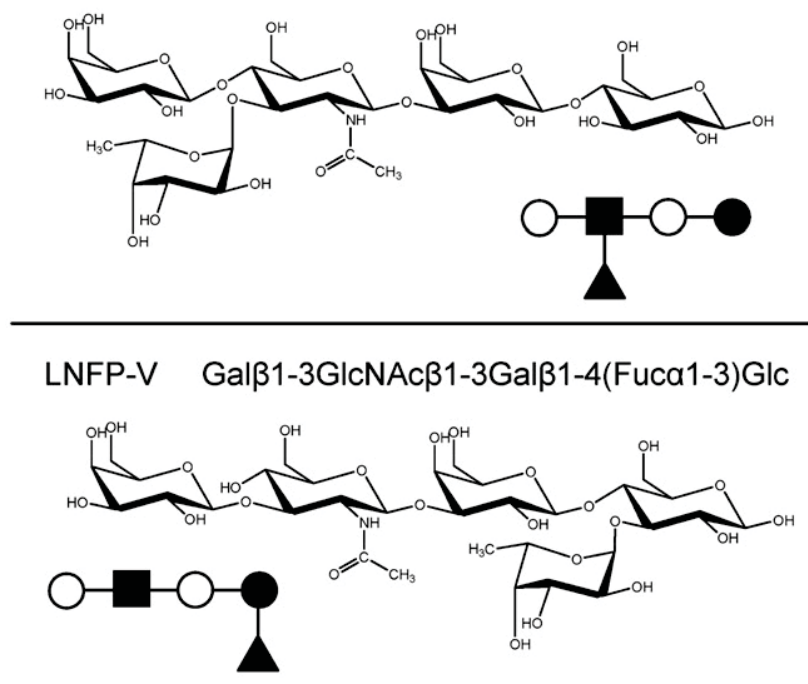

\section{LNDFH Glycans}

LNDFH-I(a) Fuca1-2Galß1-3(Fuca1-4)GIcNAcß1-3Galß1-4Glc

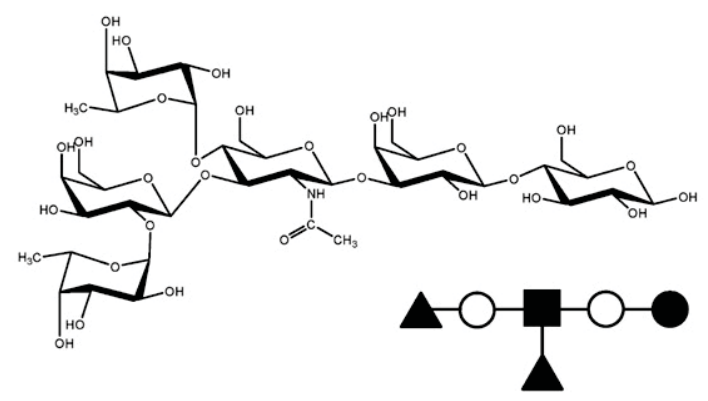

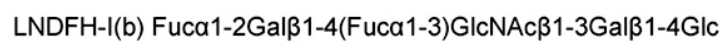

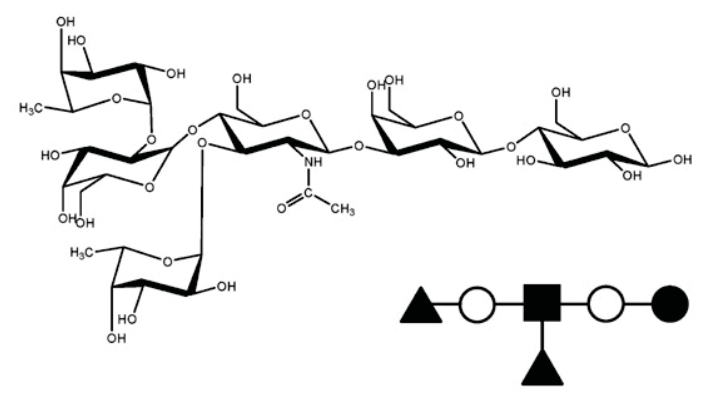

LNDFH-II Galß1-3(Fuca1-4)GIcNAcß1-3Galß1-4(Fuca1-3)Glc

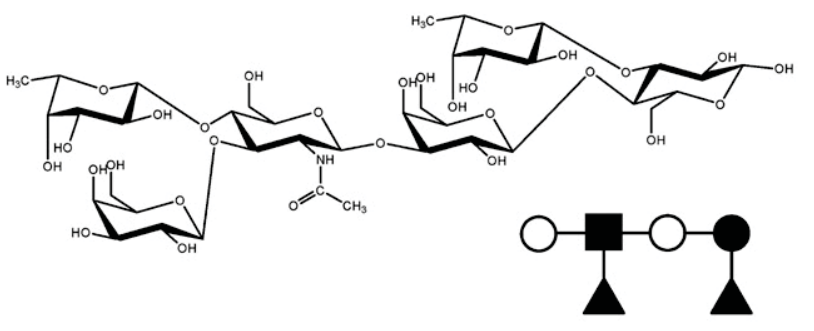

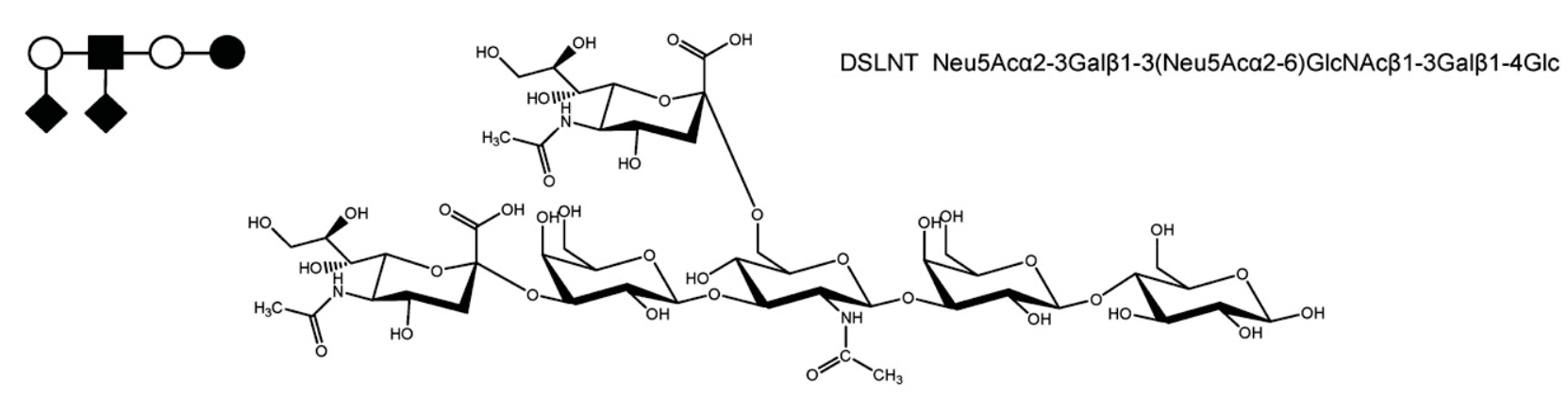

= Glc $\bigcirc=$ Gal $\quad \square=$ GlcNAc $\quad \boldsymbol{D}=$ Fuc $\quad>=\mathrm{Sia}$

Figure 1. Chemical and cartoon structures of the oligosaccharides used in the study. 
The first series, the lacto-N-fucopentaoses (LNFPs), all have the same backbone sequence but differ either in the connectivity of fucose (Fuc) or the linkage of their terminal Gal and Fuc moieties. The second series, the lacto-N-difucohexaoses (LNDFHs), also have the same backbone structure but have two Fuc residues and differ either in the connectivity or linkage of one of the Fuc residues. These series were natural choices for method development since they have been characterized by different mass spectrometric techniques previously [12, 19-21, 23, 35, 59]. For example, MALDI-MS methods have been developed that differentiate some of the LNFP isomers based on ions due to internal losses and others that reveal the interglycosidic linkage [12, $35,59]$. While several of these fragment ions facilitate isomer differentiation, they often do not provide complete sequence information and may complicate spectral interpretation of unknown oligosaccharides since they are a product of cleavage from both the reducing and nonreducing ends coupled with internal and unidentified fragments. Several methods using ESI-MS for characterization of the LNFPs and LNDFHs have also been reported. For example, Konig and Leary used metal ion coordination to alter the fragmentation pathways of the LNFPs; however, the study was focused on specific linkage determination and not sequencing [23]. Chai and coworkers used ESI-MS in conjunction with a Q-TOF mass spectrometer and identified specific "Dtype" fragments that differentiated the LNFPs and LNDFHs [21]. These fragments and several other lower mass fragments are only observed upon several stages of CAD $\left(\mathrm{MS}^{\mathrm{n}}\right)$ in the QIT [19-21]. Pfenninger and Karas developed an elaborate and comprehensive method for the isomer differentiation of oligosaccharides [19, 20] that also provides linkage information, although the resulting spectra are complicated by several different types of fragment ions.

In the present study, we report a simplified method for the sequencing of oligosaccharides in a single stage of activation by exploiting the supplementary information obtained from sequential fragmentation that is promoted by nonresonant IRMPD. Additionally, our method is further extended to an oligosaccharide with two acidic sialic acid moieties. To increase the IR absorption efficiency of the oligosaccharides, a phosphonate group was incorporated into a boronic acid that was used as a derivatizing reagent. The IR-active boronic acid (IRABA) was designed so that a nitrogen atom was adjacent to the boron atom to enhance the reactivity of the boronic acid functionality to the oligosaccharides [60]. When the IRABA is added to a solution containing a oligosaccharide of interest, the chemical reaction between the IRABA and diol functionalities of the oligosaccharide is responsible for the covalent addition of the IR-active phosphonate group. The modified analytes proved to be ideal for characterization and differentiation using IRMPD. The LNFP and LNDFH series provide a considerable challenge for differentiation and thus allow a convenient means of assessing the analytical merits of the new IRMPD method.

\section{Experimental}

\section{Chemical Reagents}

LNFP-I, II, III, V, LNDFH Ia and II, and DSLNT were purchased from V-Labs (Covington, LA). LNDFH-Ib was purchased from Sigma (St. Louis, MO). Diethyl(aminomethyl)phosphonate oxalate was purchased from Acros (Geel, Belgium). 2-formylphenylboronic acid, methanol and methylene chloride were purchased from Fisher (Somerville, NJ). All chemicals were used without further purification.

\section{Derivatization}

Stock solutions of the oligosaccharides $(500 \mu \mathrm{M})$ and the IRABA (mM) were mixed to form a 1:10 M ratio and a $5 \mu \mathrm{L}$ aliquot of $0.5 \%$ TEA was added to achieve a $\mathrm{pH}$ $\sim 9$. The reaction is reversible and a lower $\mathrm{pH}$ would result in a competition of hydrolysis of the new bonds formed. The solutions were then sonicated for $1 \mathrm{~min}$ and diluted with $0.1 \%$ TEA to $\mathrm{pH} \sim 8$ and spiked with ammonium acetate (to $0.01 \%$ ) to eliminate the domination of derivatized sodium adducts. The final solution had an oligosaccharide concentration of $10 \mu \mathrm{M}$.

\section{Mass Spectrometry}

A ThermoFinnigan LCQ Deca XP mass spectrometer (San Jose, CA) equipped with an electrospray ionization source and interfaced with a Synrad $\mathrm{CO}_{2} 50 \mathrm{~W}$ laser $(10.6 \mu \mathrm{m})$ was used for this study. The details of the system were previously described [37]. The solutions analyzed were $10 \mu \mathrm{M}$ in oligosaccharide. Typical IRMPD parameters included an irradiation time of 5 to $25 \mathrm{~ms}$ at a power of $50 \mathrm{~W}$. The helium pressure for IRMPD experiments of the derivatized oligosaccharides and comparative CAD experiments was nominally 2.8 $\times 10^{-5}$ Torr (corresponding to $\sim 1$ mTorr normally used in the quadrupole ion trap) as measured by an ionization gauge. For the underivatized oligosaccharides (i.e., without IR chromophores), the helium pressure was lowered to nominally $2.7 \times 10^{-5}$ Torr to promote the IRMPD process.

Throughout this study, the laser power was $50 \mathrm{~W}$ and the irradiation time was adjusted to tune sequential fragmentation such that the most complete structural information was obtained. At prolonged irradiation times sequential fragmentation was extensive and higher mass fragments were not observed.

\section{Synthesis of IRABA}

The procedure used was a modification of the synthesis described by Zhu and Anslyn [61]; 3.9 mmol 2formylphenylboronic acid (mM) was dissolved in 
Table 1. Monosaccharide residue masses

\begin{tabular}{lllc}
\hline \multicolumn{1}{c}{ Monosaccharide } & ID & Unknown ID & $\begin{array}{c}\text { Residue } \\
\text { mass }\end{array}$ \\
\hline \hline Fucose & Fuc & Fuc & 146 \\
Galactose & Gal & Hex & 162 \\
Glucose & Glc & Hex & 162 \\
N-acetyl-glucosamine & GlcNAc & HexNAc & 203 \\
Hexose & Hex & Hex & 162 \\
N-acetyl-hexosamine & HexNAc & HexNAc & 203 \\
Siallc acid & Sia & Sia & 219 \\
\hline
\end{tabular}

anhydrous $\mathrm{CH}_{3} \mathrm{OH}$ under argon protection; $16 \mathrm{mmol}$ $(4 \times)$ Hunig's base was added followed by $3.9 \mathrm{mmol}$ diethyl(aminomethyl)phosphonate oxalate and the solution was stirred for $16 \mathrm{~h}$ before $2.9 \mathrm{mmol} \mathrm{NaBH}_{4}$ was added slowly. The solution was stirred at room temperature for $1 \mathrm{~h}$, followed by addition of another batch of $\mathrm{NaBH}_{4}$. One $\mathrm{h}$ later, the solvent was removed under vacuum and the residue was diluted with $\mathrm{CH}_{2} \mathrm{Cl}_{2}$. The white precipitate was removed with vacuum filtration, with the filtrate subsequently concentrated. The residue was purified by flash chromatography on neutral alumina $\left(2-5 \% \mathrm{NH}_{3}\right.$-saturated $\mathrm{CH}_{3} \mathrm{OH}$ in $\left.\mathrm{CH}_{2} \mathrm{Cl}_{2}\right)$; $1 \mathrm{H} \mathrm{NMR}\left(400 \mathrm{MHz}, \mathrm{CD}_{3} \mathrm{OD}\right) \Delta$ $1.39(\mathrm{t}, \mathrm{J}=7.04,6 \mathrm{H}, \mathrm{CH} 3) \Delta 3.23(\mathrm{~d}, \mathrm{~J}=11.6,2 \mathrm{H} \mathrm{CHs})$ $\Delta 4.17$ (s, 2H CHs) $\Delta$ 4.20-4.26 (m, 4H CHs) $\Delta$ 7.15-7.43 (m, 4H CHs); 13C NMR(CD $\left.{ }_{3} \mathrm{OD}\right):=16.8$, 42.2, 43.4, 56.2, 64.4, 124.1, 127.9, 128.7, 131.4, 142.9; HRMS: calcd. $(\mathrm{M}+\mathrm{Na})^{+} 324.1148$, found 324.1135 .

\section{Results and Discussion}

The goal of this study was to develop a method for sequencing the backbone of the LNFPs and to pinpoint the sites of attachment of the fucose moieties. To allow the comprehensive evaluation of the new IRABA derivatization/IRMPD strategy for the characterization of oligosaccharides, companion CAD and IRMPD studies of the underivatized oligosaccharides were undertaken to provide benchmark comparisons. These studies are briefly described in the first section before presentation of the results for the IRABAderivatized oligosaccharides.

\section{LNFP Oligosaccharides}

The LNFP oligosaccharides studied (Figure 1) differ only in the branching position of the fucose moiety (LNFP I vs. II and III vs. V) or have the same position but differ only in the linkage (LNFP II vs. III). The oligosaccharides are characterized using the residue masses for the monosaccharide units that are listed in Table 1 and are further described using the nomenclature of Domon and Costello [62]. Since the structures of the oligosaccharides used in the study are known, it was possible to also include specific hexose identification upon analysis. In the case of an unknown, less specific identifications (unknown identifications such as
Hex instead of Gal) would be determined. The abbreviations, residue masses, and unknown identifications used are provided in Table 1.

\section{CAD and IRMPD of Deprotonated Oligosaccharides}

The CAD mass spectra of the four deprotonated oligosaccharides, $\left([\mathrm{L}-\mathrm{H}]^{-}\right)$, (Figure S1, data available as Supplementary Material section, which can be found in the electronic version of this article) and the data is tabulated in Table 2. Losses of the residue masses are labeled with the corresponding residues (e.g., loss does not include the intersaccharide oxygen atom). Losses that include the intersaccharide oxygen atom are labeled with the monosaccharide identity and an asterisk. The types of fragments observed in the CAD and IRMPD spectra (shown in Supplementary Material Figure S1 and briefly described below) are consistent with those previously reported, although the IRMPD spectra exhibit several additional sequential fragment ions. The spectra include mainly Z- or C- and B- or Y-type cleavage. The detailed fragmentation pathways of the deprotonated LNFPs have been described previously [19-21]. The CAD spectra do not display as many fragment ions as were observed in the previous studies; however the IRMPD spectra (described below) display even more. In several cases there is limited information in the first stage of fragmentation, and further analysis of these oligosaccharides using multiple sequential stages of tandem mass spectrometry is not generally viable since ion populations are too low.

The IRMPD mass spectra of the underivatized oligosaccharides were acquired to exploit the benefits of sequential fragmentation that often occur when using this nonresonant activation method. Sequential fragmentation was evident for the oligosaccharides because increases in the laser irradiation time resulted in an increase in the abundance of lower mass fragments coupled with a decrease in the abundance of higher mass fragments. For these experiments, a slightly reduced helium pressure was necessary to enhance energy accumulation at the expense of trapping efficiency (less effective collisional cooling of ions). The irradiation time was adjusted to optimize the number of informative fragment ions (see supplementary material, Figure S1). The data is tabulated in Table 2. Inspection of the IRMPD spectra indicates that the four isomers are differentiated due to the presence of ions derived from sequential fragmentation. The spectrum for deprotonated LNFP-I displays only a single loss of Hex, whereas the spectra for deprotonated LNFP-II and III display a second loss of Hex. The next sequential fragments observed for LNFP-II and III differ and are due to the loss of Hex $(\mathrm{Z} 3 \alpha / \mathrm{C} 2)$ or Fuc $(\mathrm{Z} 3 \beta / \mathrm{C} 2)$, respectively. The overall sequence of losses is consistent with the final loss 
Table 2. Underivatized LNFP glycan fragment ion abundances*

\begin{tabular}{|c|c|c|c|c|c|c|}
\hline$(M+H) * C A D$ & $\mathrm{C}_{4}{ }^{\mathrm{a}}, \mathrm{C}_{3}^{\mathrm{bc}}$ & $\mathrm{Z}_{1 \beta}$ & $\mathrm{C}_{3 \alpha}$ & $\mathrm{C}_{2}$ & $\mathrm{C}_{3 \beta} / \mathrm{C}_{2}$ & $Z_{3 \alpha}$ \\
\hline LNFP-I & 91 & - & - & - & - & - \\
\hline LNFP-II & 89 & - & - & - & - & - \\
\hline LNFP-III & 89 & - & - & - & - & - \\
\hline LNFP-V & - & 24 & 71 & - & - & - \\
\hline$(\mathrm{M}-\mathrm{H}) *$ IRMPD & $\mathrm{C}_{4}{ }^{\mathrm{a}}, \mathrm{C}_{3}{ }^{\mathrm{bc}}$ & $\mathrm{Z}_{1 \beta}$ & $\mathrm{C}_{3 \alpha}$ & $\mathrm{C}_{2}$ & $\mathrm{C}_{3 \beta} / \mathrm{C}_{2}$ & $Z_{3 \alpha}$ \\
\hline LNFP-I & 48 & - & - & - & - & - \\
\hline LNFP-II & 28 & - & - & 2 & 2 & 32 \\
\hline LNFP-III & 29 & - & - & 2 & 35 & - \\
\hline LNFP-V & - & 11 & 49 & - & - & - \\
\hline$(\mathrm{M}+\mathrm{Na})^{*} \mathrm{CAD}$ & $\mathrm{Y}_{4}{ }^{\mathrm{a}}$ or $\mathrm{Y}_{3}^{\mathrm{bc}}$ & $\mathrm{B}_{4}$ & $\mathrm{Y}_{4} / \mathrm{B}_{4}{ }^{\mathrm{a}}, \mathrm{Y}_{3 \beta} / \mathrm{B}_{3}{ }^{\mathrm{bc}}, \mathrm{B}_{3}{ }^{\mathrm{d}}$ & $\mathrm{Y}_{4} / \mathrm{B}_{3}, \mathrm{Y}_{3 \beta} / \mathrm{B}_{2}, \mathrm{~B}_{2}$ & $X_{2}^{2,3}$ & $x_{3}^{2,4}$ \\
\hline LNFP-I & 83 & 6 & 3 & -4 & - & - \\
\hline LNFP-II & 68 & 14 & 7 & - & - & - \\
\hline LNFP-III & 68 & 14 & 5 & - & - & - \\
\hline LNFP-V & 83 & & 3 & - & - & - \\
\hline$(\mathrm{M}+\mathrm{Na})^{*}$ IRMPD & $Y_{4}{ }^{a}$ or $Y_{3}{ }^{b c}$ & $\mathrm{~B}_{4}$ & $\mathrm{Y}_{4} / \mathrm{B}_{4}{ }^{\mathrm{a}}, \mathrm{Y}_{3 \beta} / \mathrm{B}_{3}{ }^{\mathrm{bc}}, \mathrm{B}_{3}{ }^{\mathrm{d}}$ & $\mathrm{Y}_{2} / \mathrm{B}_{4}$ & $X_{2}^{2,3}$ & $X_{3}^{2,4}$ \\
\hline LNFP-I & 35 & -4 & 2 & - & 13 & 11 \\
\hline LNFP-II & 40 & - & 4 & 12 & - & - \\
\hline LNFP-III & 34 & - & 4 & 20 & - & - \\
\hline LNFP-V & 49 & - & 2 & 2 & - & - \\
\hline
\end{tabular}

*As a percentage of total sequence and parent ion abundances, $+/-5 \%$.

aLNFP-I.

bLNFP-II.

'LNFP-III.

dLNFP-V.

being identified as the monosaccharide unit that is linked to the GlcNAc for each of these isomers (Gal for LNFP-II and Fuc for LNFP-III), indicating that loss of the terminal $1 \rightarrow 3$ linked monosaccharides is a much more facile loss than the loss of the terminal $1 \rightarrow 4$ linked monosaccharides. The IRMPD spectrum for LNFP-V is unique in that there are two types of cleavages involving Fuc; the first being the loss of Fuc $\left(Z_{1 \beta}\right)$ and another the loss of Fuc in conjunction with a loss of Hex $\left(\mathrm{C}_{3 \alpha}\right)$. Examination of the LNFP structures suggests that the first losses from the nonreducing end generally occur as a Z-type cleavage and losses from the reducing end occur as a C-type cleavage. Unfortunately, for each isomer there is a lack of a significant amount of information for sequencing the backbone and Fuc position. In addition, the sequential losses are limited to one to three monosaccharide units, thus restricting the sequence coverage. Overall, the CAD and IRMPD spectra of the deprotonated oligosaccharides display losses that cannot be immediately identified as occurring from the reducing or nonreducing end of the oligosaccharides. Additionally, some ions (e.g., 348 and 364 Da in Figure S1f and S1g, respectively) cannot be confidently identified as being a result of a sequential loss or an internal loss (e.g., "D-type loss"). Even with the correct identification of losses, it would require several stages of dissociation to completely characterize the oligosaccharides. In general, neither the negative ion mode CAD nor IRMPD spectra could provide enough information to sequence these isomers with confidence.

\section{CAD and IRMPD of Sodium-Cationized Oligosaccharides}

Due to the unsatisfactory structural characterization of the deprotonated oligosaccharides, the CAD and IRMPD spectra of the sodium-cationized oligosaccharides were obtained for comparison. In the positive ion mode, sodium-cationized complexes were formed almost exclusively over the protonated species, and thus they were used for the analyses. The types of losses observed for the sodium complexes were similar to those observed for the deprotonated oligosaccharides (supplemental Figure S1). The residue losses observed for all isomers are tabulated in Table 2. The CAD spectra are all highlighted by a predominant loss of Fuc, rendering these spectra uninformative.

The IRMPD spectra are highlighted by a loss of Fuc followed by a sequential loss of $\mathrm{Hex}^{*}$, the asterisk indicating the inclusion of the intersaccharide oxygen in the neutral loss. Following the Hex* loss, the LNFP-I isomer undergoes cross-ring cleavages of the type $X_{3}[2$, $4]$ and $X_{3}[2,3]$, using the nomenclature of Domon and Costello [62], while the other isomers undergo an additional Hex loss. Inspection of the data summarized in Table 2 reveals why tandem mass spectrometric analysis of the sodium-cationized oligosaccharides suffers from the same general limitation as that observed for the deprotonated oligosaccharides in that there is limited structural or sequence information provided in any of the spectra. Since losses observed in the spectra cannot be immediately identified as occurring from the reducing or nonreducing end of the oligosaccharides, it is difficult to obtain confident sequence data. It would 


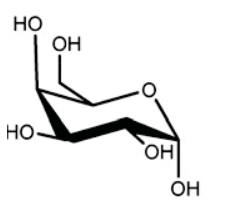

Glc

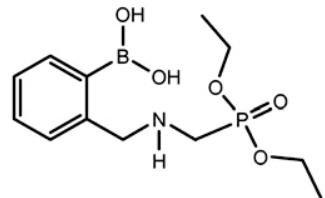

IRABA

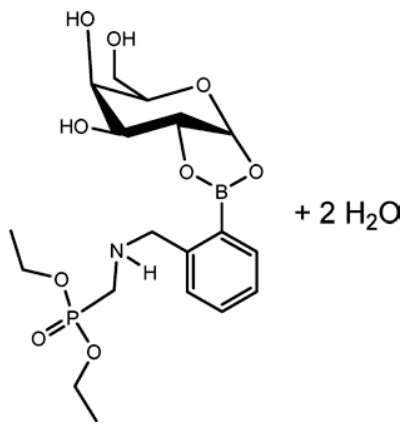

Scheme 1

require several stages of dissociation for more complete structural information which is unfeasible with the low ion populations obtained for the sodium-cationized oligosaccharides. Since neither the CAD nor the IRMPD spectra of the underivatized LNFPs yielded diagnostic fragment ions or sufficient sequencing information for the isomers, the boronic acid derivatization strategy was explored next.

\section{Derivatized LNFP Oligosaccharides}

We envisioned a special boronic acid derivatization reagent that would react efficiently with the oligosaccharides and would incorporate a strong IR chromophore. The IR-active boronic acid (IRABA) shown in Scheme 1 was designed to meet these criteria. The strategically placed nitrogen atom adjacent to the boronic acid functionality accelerates the derivatization reaction [60]. Without the nitrogen atom, the reaction with oligosaccharides proceeds with low efficiency. The phosphonate group provides an excellent IR chromophore at $10.6 \mu \mathrm{m}$. Simple addition of the IRABA ligand to the oligosaccharide solutions results in formation of two covalent bonds between the boronic acid and the oligosaccharides in conjunction with two water losses. An example of this type of reaction for Glc is detailed in Scheme 1. Typical ESI mass spectra for the IRABA-derivatized LNFP-I reaction mixture are depicted in Figure 2 in both the negative and positive ion modes. In the negative ESI mode, the abundance of the IRABA-derivative is greater than that of the deprotonated oligosaccharide. In addition, there is a product attributed to the derivatized oligosaccharide with a loss of $28 \mathrm{Da}$, corresponding to a loss of ethylene from the diethylphosphonate functionality. In the positive ESI mass spectra, the IRABA-derivatized and sodiumcationized oligosaccharides are observed. Yields of the derivatized products are high, and sample clean-up is unnecessary. Following successful formation of the derivatized oligosaccharides, the diagnostic value of the fragmentation patterns obtained by CAD or IRMPD was evaluated.

\section{CAD and IRMPD of Derivatized Oligosaccharides}

The CAD and IRMPD data for the IRABA-derivatized LNFPs in the negative ion mode were collected by activation of the [LNFPI + IRABA $\left.-2 \mathrm{H}_{2} \mathrm{O}-\mathrm{H}\right]^{-}$ species, and the resulting spectral data tabulated in Table 3. While the fragment ions in the IRMPD spectra of the underivatized oligosaccharides are a result of cleavages from both the reducing and nonreducing ends of the oligosaccharides, the most prominent fragment ions in the IRMPD spectra of the derivatized analogs are a result of cleavages from the nonreducing end.

The CAD spectra for the derivatized LNFPs of the type $\left[\mathrm{LNFP}+\mathrm{IRABA}-2 \mathrm{H}_{2} \mathrm{O}+\mathrm{H}\right]^{+}$are shown in

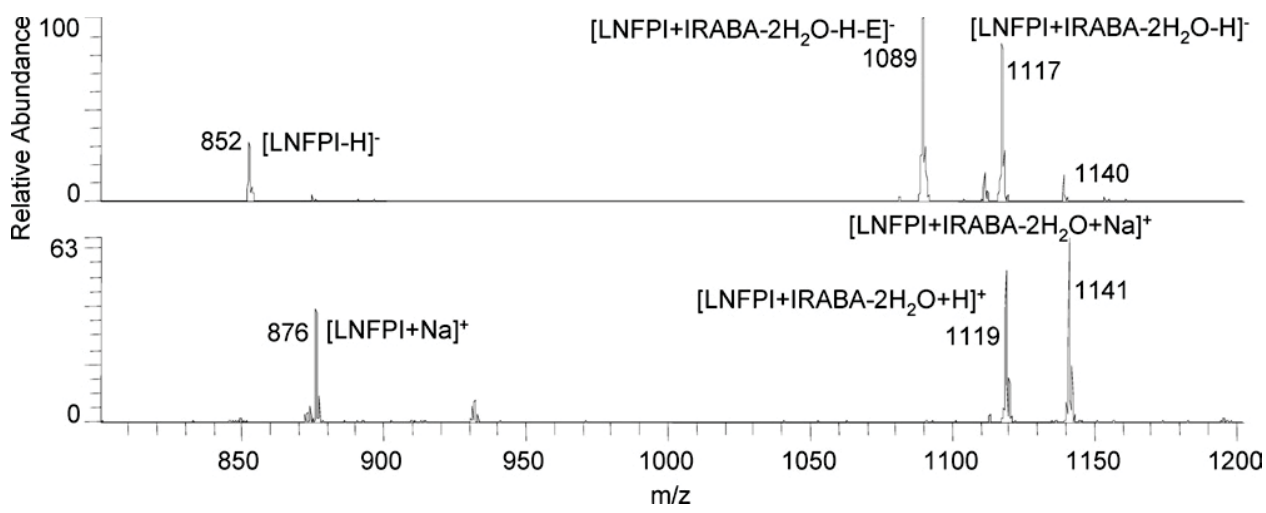

Figure 2. Full scan spectra of solutions of LNFPI after derivatization with the IRABA: (a) negative ion mode, (b) positive ion mode. " $\mathrm{E}$ " indicates the loss of ethene. 
Table 3. Derivatized LNFP glycan fragment ion abundances ${ }^{a}$

\begin{tabular}{|c|c|c|c|c|c|c|c|c|c|}
\hline$\left[\text { LNFP }+ \text { IRABA }-2 \mathrm{H}_{2} \mathrm{O}-\mathrm{H}\right]^{*}$ CAD & $\mathrm{C}_{4}, \mathrm{C}_{3}$ & $\mathrm{C}_{3}$ & & & & & & & \\
\hline LNFP-I & 60 & - & & & & & & & \\
\hline LNFP-II & 79 & - & & & & & & & \\
\hline LNFP-III & 50 & - & & & & & & & \\
\hline LNFP-V & - & 86 & & & & & & & \\
\hline$\left[\text { LNFP }+ \text { IRABA }-2 \mathrm{H}_{2} \mathrm{O}-\mathrm{H}\right]^{*}$ IRMPD & $\mathrm{C}_{4}, \mathrm{C}_{3}$ & $\mathrm{C}_{2}$ & $\mathrm{C}_{3 \alpha}$ & & & & & & \\
\hline LNFP-I & 42 & 3 & - & & & & & & \\
\hline LNFP-II & 30 & 1 & - & & & & & & \\
\hline LNFP-III & 17 & - & - & & & & & & \\
\hline LNFP-V & - & - & 45 & & & & & & \\
\hline$\left[\right.$ LNFP + IRABA $\left.-2 \mathrm{H}_{2} \mathrm{O}+\mathrm{H}\right] *$ CAD & $Y_{4}, Y_{3 \beta}, Y_{1 \beta}$ & $\mathrm{Y}_{3}$ & $\mathrm{~B}_{2}, \mathrm{~B}_{2}$ & $\mathrm{Y}_{2 \alpha}$ & $\mathrm{B}_{2}$ & $\mathrm{Y}_{2}$ & $Y_{2}, Y_{2 \alpha} / Y_{1 \beta}$ & $\mathrm{B}_{1}$ & $\mathrm{Y}_{0}$ \\
\hline $\begin{array}{l}\text { LINFP-I } \\
\text { LNFP-II }\end{array}$ & 18 & - & 43 & - & $\begin{array}{l}4 \\
4\end{array}$ & - & 31 & - & - \\
\hline LNFP-III & 31 & - & 52 & - & 2 & - & 10 & - & - \\
\hline LNFP-V & 47 & - & & 15 & 29 & - & 3 & - & - \\
\hline$\left[\text { LNFP }+ \text { IRABA }-2 \mathrm{H}_{2} \mathrm{O}+\mathrm{H}\right]^{*}$ IRMPD & $\mathrm{Y}_{4}, \mathrm{Y}_{3 \beta}, \mathrm{Y}_{1 \beta}$ & $Y_{3}$ & $\mathrm{~B}_{3}, \mathrm{~B}_{2}$ & $Y_{2 u}$ & $\mathrm{~B}_{2}$ & $\mathrm{Y}_{2}$ & $Y_{1}$ & $\mathrm{~B}_{1}$ & $Y_{0}$ \\
\hline LNFP-I & 4 & 6 & - & - & 3 & 20 & 10 & 8 & 17 \\
\hline LNFP-II & 6 & - & 2 & - & 7 & 25 & 14 & 5 & 8 \\
\hline LNFP-III & 12 & - & 2 & - & 5 & 23 & 10 & 2 & 17 \\
\hline LNFP-V & 15 & - & - & 5 & 3 & 17 & 17 & 6 & 13 \\
\hline
\end{tabular}

${ }^{\mathrm{a}} \mathrm{As}$ a percentage of total sequence and parent ion abundances, $+/-5 \%$.

Figure 3, and the complete series of data is tabulated in Table 3. The data suggest that there are two general dissociation pathways: the most prominent one from sequential losses from the nonreducing end of the oligosaccharides (a result of Y-type cleavages) and the other entailing losses from the reducing end (a result of
B-type cleavages). In addition, there are also losses of the entire phosphonate moiety from the precursor ions, and these are indicated with a filled circle in the spectra. The first general type of pathway is defined by sequential losses from the nonreducing end of the oligosaccharides (losses highlighted in bold type) and corresponds

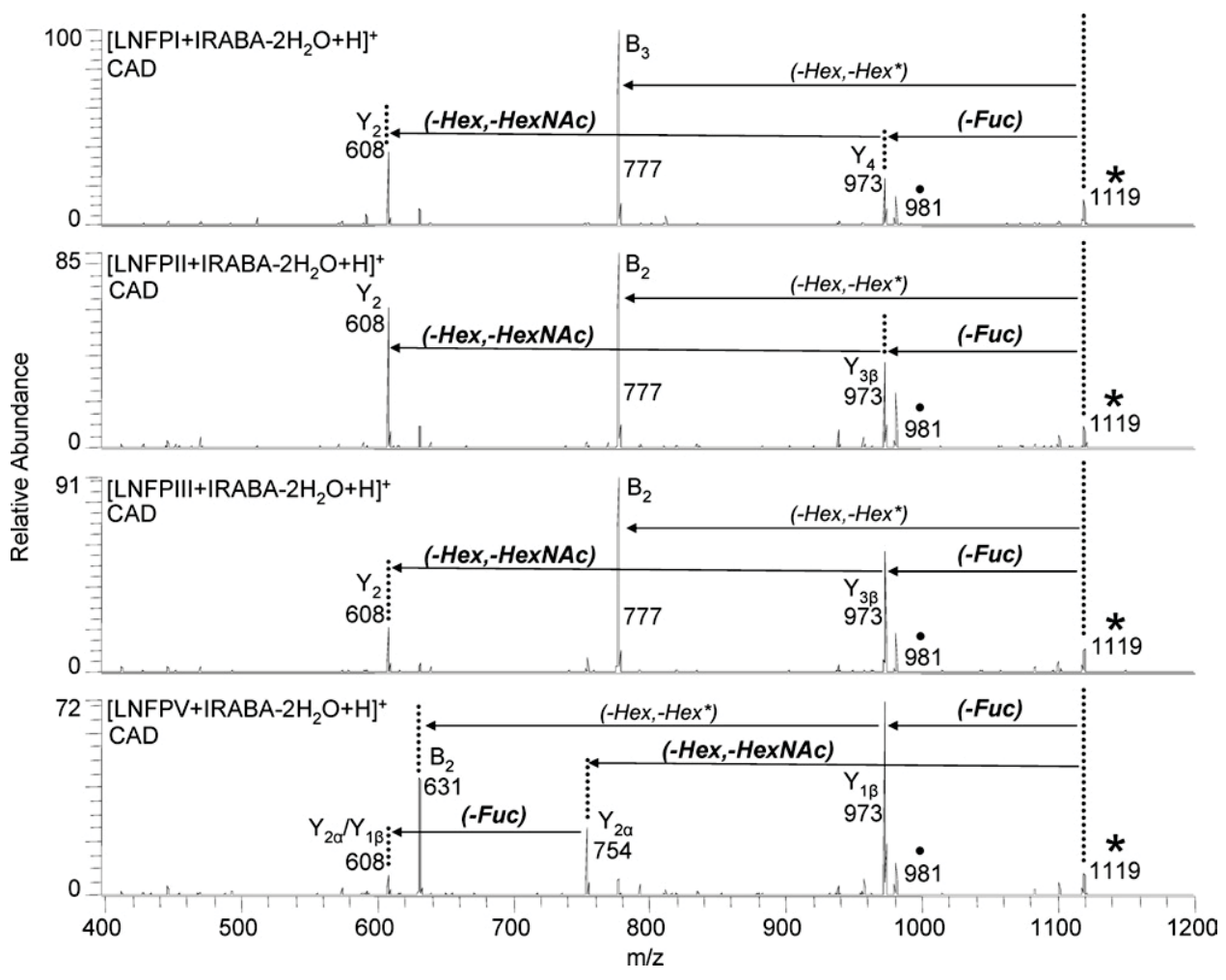

Figure 3. Positive ion mode $\mathrm{CAD}$ spectra of oligosaccharides after derivatization with IRABA: (a) $\left[\right.$ LNFPI + IRABA $-2 \mathrm{H}_{2} \mathrm{O}+\mathrm{H}^{+}$, (b) $\left[\text {LNFPII + IRABA }-2 \mathrm{H}_{2} \mathrm{O}+\mathrm{H}\right]^{+}$, (c) [LNFPIII + IRABA $\left.2 \mathrm{H}_{2} \mathrm{O}+\mathrm{H}\right]^{+}$, (d) $\left[\mathrm{LNFPV}+\mathrm{IRABA}-2 \mathrm{H}_{2} \mathrm{O}+\mathrm{H}\right]^{+}$. Fragments ions that stem from loss of a phosphonate moiety are indicated with a filled circle. 


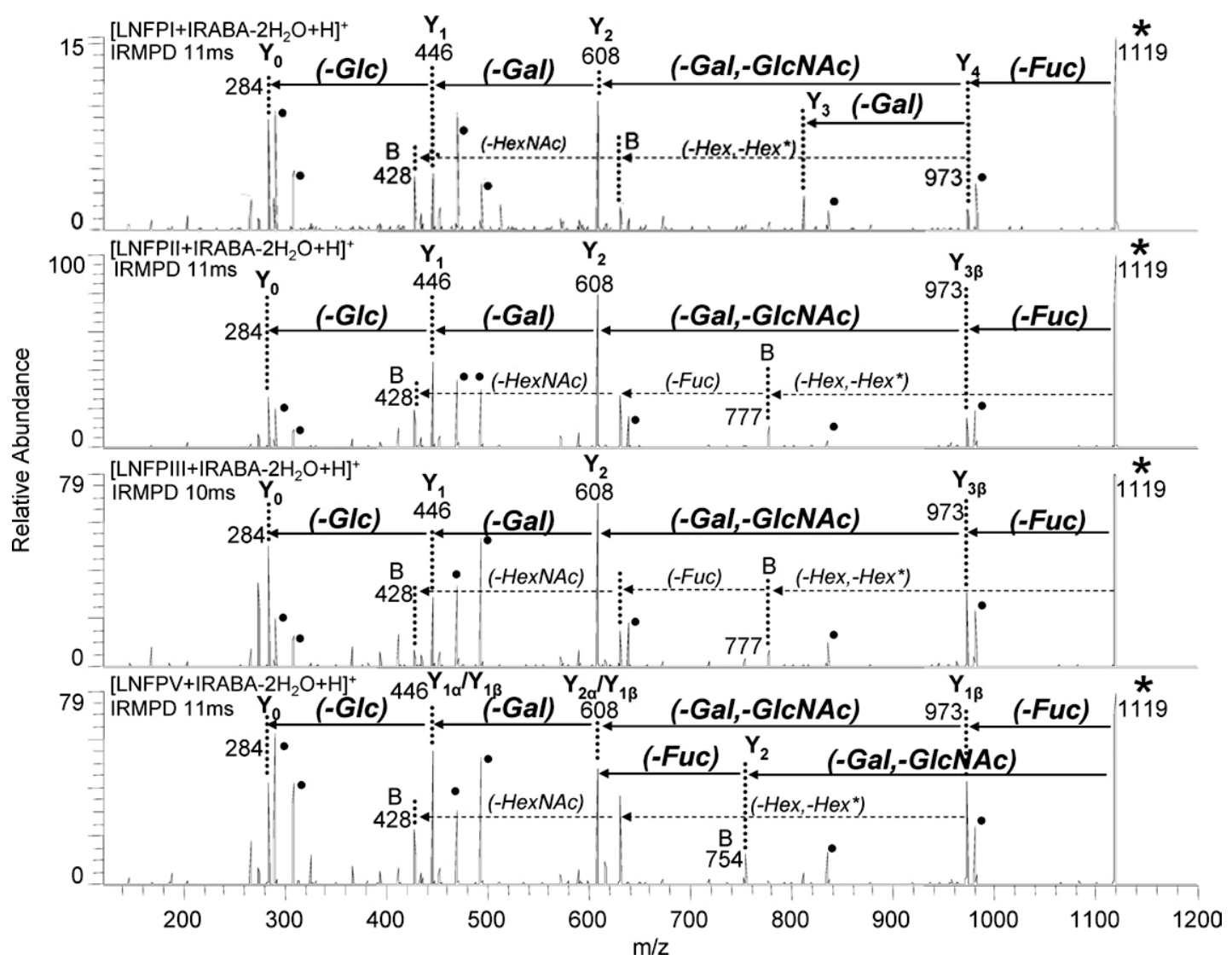

Figure 4. IRMPD spectra of LNFP oligosaccharides after derivatization with IRABA: (a) [LNFPI + IRABA $-2 \mathrm{H}_{2} \mathrm{O}+\mathrm{H}^{+}$, (b) $\left[\text {LNFPII + IRABA }-2 \mathrm{H}_{2} \mathrm{O}+\mathrm{H}^{+} \text {, (c) [LNFPIII + IRABA }-2 \mathrm{H}_{2} \mathrm{O}+\mathrm{H}\right]^{+}$, (d) $\left[\mathrm{LNFPV}+\mathrm{IRAB}-2 \mathrm{H}_{2} \mathrm{O}+\mathrm{H}\right]^{+}$. Fragments ions that stem from loss of a phosphonate moiety are indicated with a filled circle.

to a loss of Fuc and a combined loss of Hex and HexNAc for the LNFPs. The persistent combined loss of Hex and HexNAc suggests that cleavage at the nonreducing end of the GlcNAc is not a facile process. The LNFP-V isomer, with the Fuc attached to the reducing Glc, is differentiated from the other three by the initial loss of combined Hex and HexNAc, which is observed as a prominent fragment ion of $\mathrm{m} / \mathrm{z} 754 \mathrm{Da}$ in the spectrum. Since the Fuc moiety associated with LNFP-I is the terminal saccharide at the nonreducing end, an initial loss of Hex, HexNAc could only occur via an internal loss, a type of cleavage not observed with the oligosaccharides studied using our derivatization strategy. For the LNFP-II and III isomers, both the Gal and Fuc are attached to the GlcNAc, which also makes this fragment much less likely. The second general pathway observed in Figure 3 involves the combined loss of Hex $\mathrm{Hex}^{*}$. Since fragmentation occurs from both the reducing and nonreducing ends, in most cases there is no indication from which end the sequential residue losses stem. Hex Hex loss is consistent for all of the other compounds and arises from cleavage at the reducing end of the oligosaccharide. In addition, the spectral data suggests that the inclusion of the oxygen atom in the fragments in this type of pathway is characteristic for losses initiated from the reducing end of the oligosaccharides for the IRABA-derivatized species. While these CAD spectra do include some diagnostic structural information and allow limited isomer differentiation, the sequence coverage of the oligosaccharides is minimal. It may be possible to obtain more structural information by performing further stages of CAD; however, multiple stages are frequently not feasible since the ion populations for oligosaccharides are generally not sufficient for these experiments. For these reasons, IRMPD was employed so that the sequential fragmentation that is commonly promoted by the nonresonant nature of IR photoactivation may be exploited for characterization of the oligosaccharides.

The IRMPD spectra for the IRABA derivatized LNFPs are depicted in Figure 4, and the entire series of mass spectral data is tabulated in Table 3. As with the CAD spectra, there are two general fragmentation pathways observed. The most prominent pathway (bold type) results from sequential cleavages from the nonreducing end of the oligosaccharides. The secondary pathway results from cleavages that begin at the reducing end of the oligosaccharides and the series of losses include the intersaccharide oxygen atom that is indicative of cleav- 
ages from the reducing end of the IRABA derivatized oligosaccharides.

In addition to the same losses observed in the CAD spectra, the IRMPD spectra also reveal ions that have undergone subsequent dissociation. These secondary IRMPD events are responsible for the two additional Hex losses observed for all of the LNFPs in Figure 4 (-Gal, $446 \mathrm{Da}$ and -Glc, $284 \mathrm{Da}$ ). As a result, there is complete sequence coverage for each of the LNFPs. In addition, the diagnostic pathway for the LNFP-V is present (initial loss of Gal + GlcNAc) along with a diagnostic ion for the LNFP-I isomer that was not observed in the CAD spectra $\left(\mathrm{Y}_{3}\right)$. It is surmised that this ion is due to sequential fragmentation of the fragment ion at 973 Da due to the initial loss of Fuc. However, there is still not a single diagnostic ion that differentiates the LNFP-II and III isomers. These two isomers only differ in the reversed linkage positions of their nonreducing terminal Fuc and Gal moieties.

The primary fragmentation pathway (i.e., the sequence of losses initiated from the nonreducing end) is the key to reliable sequencing of the oligosaccharides. Since the fragments that arise from this pathway only stem from the nonreducing end of the oligosaccharide, the resulting sequential losses (masses of individual residues) map out the structure of the oligosaccharides. In contrast, the secondary pathways include cleavages from both ends of the oligosaccharides and therefore do not reliably map the structures. This primary fragmentation pathway is observed only when the oligosaccharides are derivatized. Without derivatization, there are mixtures of losses that define the different types of secondary pathways with losses occurring from both the reducing and nonreducing ends of the oligosaccharides. Therefore, derivatization coupled with IRMPD has proven to be an excellent technique for sequencing the LNFPs in one activation event.

\section{Site of Derivatization}

The nature of the neutral losses related to the primary fragmentation pathway in the IRMPD spectra suggests that the IRABA is attached to the reducing sugar. Since the reducing sugar is the most reactive and may have both the $\alpha$ and $\beta$ configuration, it may bear the cis-diol functionality that readily reacts with boronic acids. The existence of the secondary pathway suggests that there is at least one other site of derivatization. Since the first losses in the secondary pathway include two Hex and Fuc, it is surmised that the secondary site of attachment is at one of the Gal moieties that also possess the cis-diol functionality.

To further elucidate the site of attachment, the reducing end of the LNFP-II isomer was reduced with sodium borohydride. The reduction converts the aldehyde of the reducing sugar to an alcohol forming an alditol. After the reduction reaction, the mixture was cleaned up using a Carbograph SPE cartridge (Alltech, Deerfield, IL), dried and rediluted before ESI-MS anal- ysis. The resulting ESI mass spectra of the reaction product indicates complete conversion to the alditol since there is a prominent ion at $\mathrm{m} / \mathrm{z} 878$ corresponding to [LNFP $-\mathrm{II}($ alditol $)+\mathrm{Na}]^{+}$. In contrast, the ESI mass spectra of the oligosaccharide after the reduction reaction did not display an ion due to any unreduced species. The alditol form of LNFP-II was then reacted with the IRABA ligand, and no IRABA derivatization products were observed in the spectra, thus indicating that LNFP-II in the reduced form did not react with the IRABA reagent. Given these results, it is surmised that the IRABA reacts primarily at the reducing sugar and that any other sites of derivatization are minor products.

\section{LNDFH Oligosaccharides}

Since the derivatization of the LNFP series with the IRABA ligand followed by IRMPD was such an effective structural characterization strategy, the method was expanded to another series of oligosaccharides, the LNDFH series shown in Figure 1. The IRMPD spectra of these IRABA derivatized oligosaccharides indicate that they dissociate by the same two pathways that were observed for the LNFP series (Figure 5). This data is tabulated in Table 4. As with the LNFP series, the primary fragmentation pathway consists of cleavages of one type that take place sequentially from the nonreducing to the reducing end.

The primary fragmentation pathway provides detailed sequence information for the LNDFH oligosaccharides with the exception of the two isomers that only differ in their reversed Gal and Fuc linkages to the central GlcNAc (Ia and Ib). Following the loss of terminal or branching Fuc moieties, fragmentation of the backbone for each of the isomers from the nonreducing end starts with the combined Gal-GlcNAc losses followed by two sequential Hex losses (Gal and Glc). Further, it is evident from the IRMPD spectra of LNDFH-Ia and $\mathrm{Ib}$ that there is a Fuc attached to both the nonreducing terminal Gal and the GlcNAc since there is an initial Fuc loss followed by a combined Fuc-GalGlcNAc loss. This result further confirms the sequential nature of the losses that correspond to the primary pathway. Other possible alternative sequences of these oligosaccharides, Fuc-(Fuc)-HexNAc-Hex- or Fuc-HexNAc(Fuc)-Hex-, are discounted because in each of the IRMPD spectra throughout this study there has been a key intersaccharide cleavage at the reducing end of GlcNAc that would yield characteristic ions for Fuc(Fuc)-HexNAc and Fuc-HexNAc, respectively. These fragments ions are not observed in the IRMPD mass spectra, thus discrediting these alternative sequences. Therefore, the two Fuc must reside on the nonreducing ends Gal and GlcNAc.

The difference in the IRMPD spectrum for the LNDFH-II isomer is a result of one Fuc having a different position. Since the initial Fuc loss precedes the Gal-GlcNAc combined loss, it is evident that this Fuc 


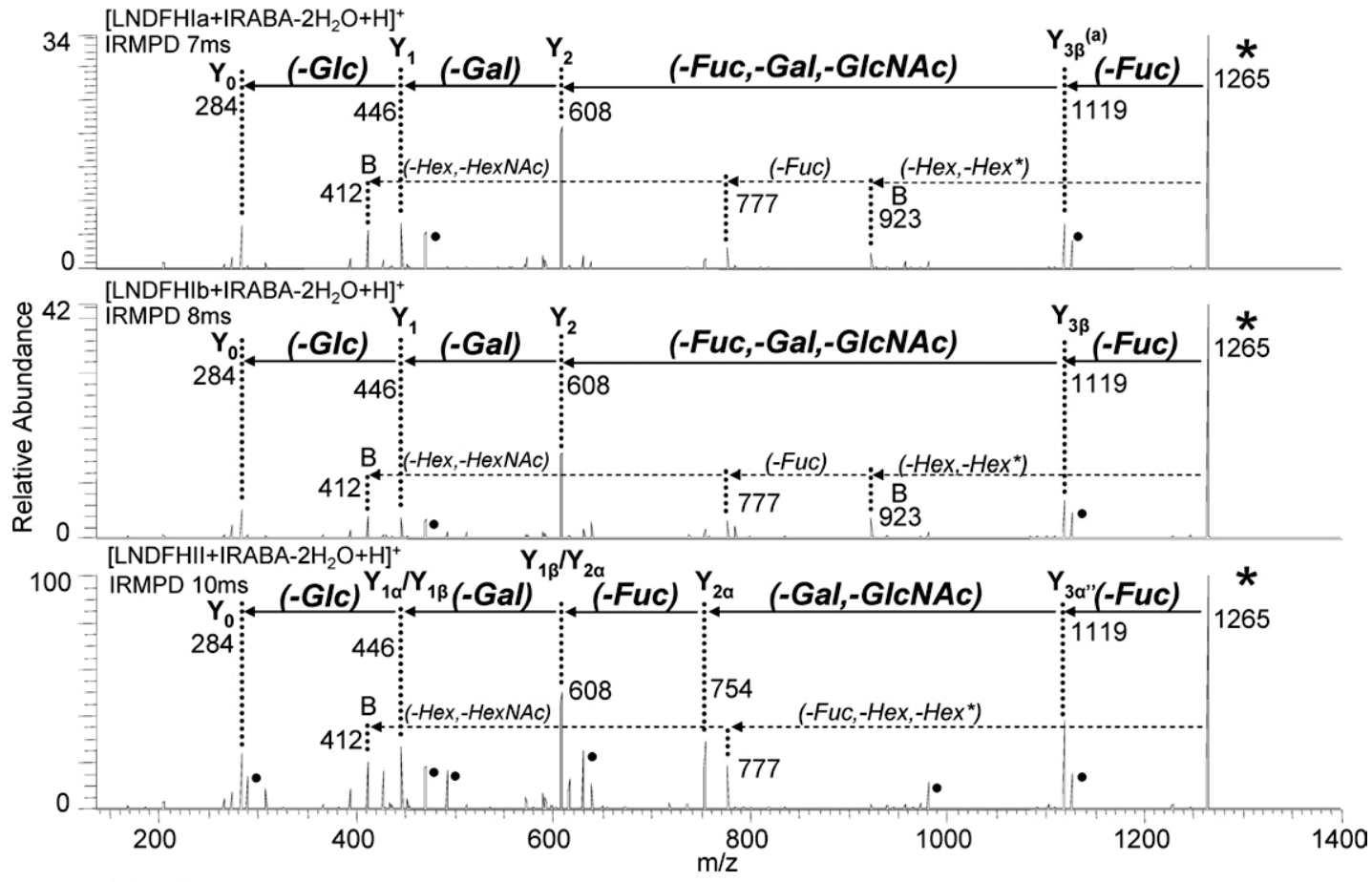

(a) or $\mathrm{Y}_{4 \alpha}$

Figure 5. IRMPD spectra of LNDFH oligosaccharides after derivatization with IRABA: (a) [LNDF$\left.\mathrm{HIa}+\mathrm{IRABA}-2 \mathrm{H}_{2} \mathrm{O}+\mathrm{H}\right]^{+}$, (b) $\left[\mathrm{LNDFHIb}+\mathrm{IRABA}-2 \mathrm{H}_{2} \mathrm{O}+\mathrm{H}\right]^{+}$, (c) [LNDFHII + IRABA $\left.2 \mathrm{H}_{2} \mathrm{O}+\mathrm{H}\right]^{+}$. Fragments ions that stem from loss of a phosphonate moiety are indicated with a filled circle.

resides on either the Gal or GlcNAc. However, if the Fuc residue were attached to the terminal Gal, one would expect an ion of $\mathrm{m} / \mathrm{z} 957$ that corresponds to a loss of Gal following the loss of Fuc. The LNFP-I isomer has this type of Fuc-Gal linkage and the corresponding fragment ion was observed in its IRMPD spectrum (Figure $4, m / z$ 811). Therefore, the initial Fuc loss must be attached to the GlcNAc. With only two Hex left on the backbone, the remaining Fuc must be attached to one or the other. Since this Fuc loss precedes the loss of both of these Hex moieties, it could be attached to either one. If the sequence continues beyond these two Hex moieties, there may be more fragment ions observed that would make it possible to pinpoint the precise location of the remaining Fuc.

\section{DSLNT Oligosaccharide}

The IRABA derivatization strategy was further extended to an acidic hexasaccharide, disialyllacto-N-tetraose (DSLNT), shown in Figure 1. As observed with the previous oligosaccharide series, the principal fragments

Table 4. Derivatized LNDFH glycan fragment ion abundances ${ }^{a}$

\begin{tabular}{|c|c|c|c|c|c|c|c|}
\hline$\left[\mathrm{LNDFH}+\mathrm{IRABA}-2 \mathrm{H}_{2} \mathrm{O}-\mathrm{H}\right]^{*} \mathrm{CAD}$ & $\mathrm{C}_{4}$ & $\mathrm{Z}_{1 \beta}$ & & & & & \\
\hline LNDFH-la & 86 & - & & & & & \\
\hline LNDFH-Ib & 80 & - & & & & & \\
\hline LNDFH-II & - & 98 & & & & & \\
\hline$\left[\mathrm{LNDFH}+\mathrm{IRABA}-2 \mathrm{H}_{2} \mathrm{O}-\mathrm{H}\right]^{*}$ IRMPD & $\mathrm{C}_{4}$ & $\mathrm{C}_{4} / \mathrm{Y}_{3 \beta} / \mathrm{Y}_{3 \alpha}$ & $\mathrm{Y}_{3 \alpha}{ }^{*} / \mathrm{Y}_{3 \alpha}{ }^{*}$ & & & & \\
\hline LNDFH-la & 17 & 40 & - & & & & \\
\hline LNDFH-Ib & 12 & 49 & - & & & & \\
\hline LNDFH-II & - & - & 56 & & & & \\
\hline$\left[\text { LNDFH + IRABA }-2 \mathrm{H}_{2} \mathrm{O}+\mathrm{H}\right]^{*} \mathrm{CAD}$ & $\mathrm{Y}_{3 \beta}, \mathrm{Y}_{3 \alpha}{ }^{*}$ & $\mathrm{~B}_{3}$ & $\mathrm{~B}_{2}, \mathrm{~B}_{3} / \mathrm{Y}_{3 \beta}$ & $\mathrm{Y}_{2 \alpha}$ & $Y_{2}, Y_{1 \beta} / Y_{2 \alpha}$ & & \\
\hline LNDFH-la & 31 & 35 & 8 & - & 24 & & \\
\hline LNDFH-Ib & 34 & 44 & 4 & - & 11 & & \\
\hline LNDFH-II & 29 & - & 23 & 24 & 5 & & \\
\hline $\begin{array}{l}\left.\text { [LNDFH + IRABA }-2 \mathrm{H}_{2} \mathrm{O}+\mathrm{H}\right]^{*} \text { IRMPD } \\
\text { LNDFH-la }\end{array}$ & $\mathrm{Y}_{3 \beta^{\prime}}, \mathrm{Y}_{3 x}$ & $\begin{array}{c}\mathrm{B}_{3} \\
3\end{array}$ & $\begin{array}{c}\mathrm{B}_{2}, \mathrm{~B}_{3} / \mathrm{Y}_{3 \beta} \\
10\end{array}$ & $\mathrm{Y}_{2 u}$ & $\begin{array}{c}Y_{2}, Y_{1 \beta} / Y_{2 u} \\
61\end{array}$ & $\begin{array}{c}\mathrm{Y}_{1}, \mathrm{Y}_{\mathrm{u}} / \mathrm{Y}_{1 \beta} \\
19\end{array}$ & $\begin{array}{l}Y_{0} \\
19\end{array}$ \\
\hline LNDFH-Ib & 16 & 6 & 6 & & 19 & 6 & 13 \\
\hline LNDFH-II & 39 & & 7 & 29 & 17 & 9 & 8 \\
\hline
\end{tabular}

${ }^{\mathrm{a} A s}$ a percentage of total sequence and parent ion abundances, $+/-5 \%$. 


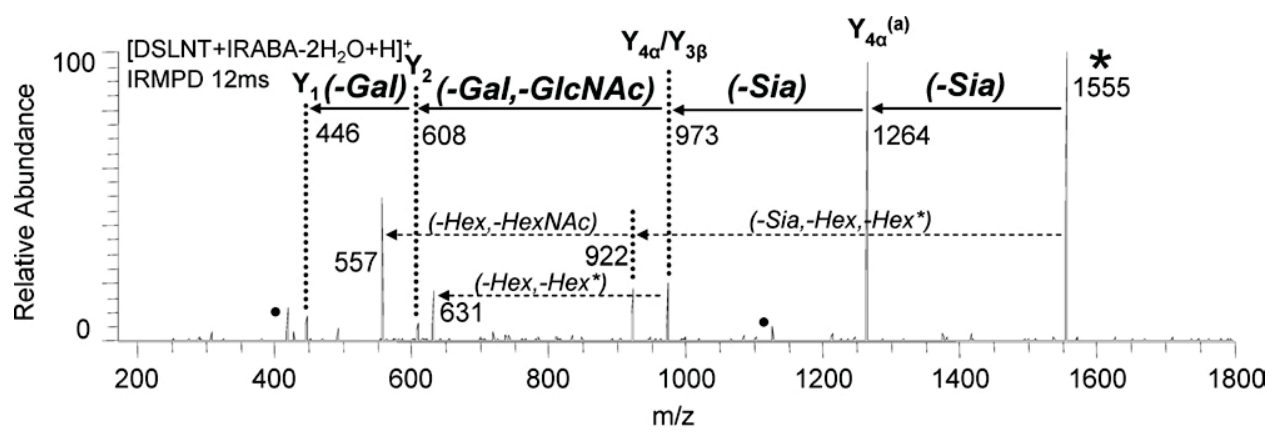

(a) or $Y_{3 \beta}$

Figure 6. IRMPD spectrum of DSLNT after derivatization with IRABA. Fragments ions that stem from loss of a phosphonate moiety are indicated with a filled circle.

are a series that arise from the backbone from the nonreducing to reducing end of the oligosaccharide (Figure 6). Following the loss of the two Sia moieties, a combined loss of Gal-GlcNAc resulting from cleavage at the reducing end of GlcNAc indicates the sequence Sia-Gal-(Sia)-GlcNAc. Subsequent losses reveal the completion of the sequence Gal-Glc. The successful sequencing of this oligosaccharide indicates that the method may be further applied to larger and different types of oligosaccharides.

\section{Conclusions}

The sequencing of oligosaccharides has been simplified by the use of IRMPD in a QIT. This was accomplished with the use of a boronic acid derivatizing reagent that was also functionalized with an IR-active phosphonate group to facilitate the photon absorption process. The oligosaccharides underwent modification by simple addition of the IRABA with reaction times of $\sim 1 \mathrm{~min}$ and did not require sample cleanup before analysis by ESI-MS. The oligosaccharide products dissociated with high efficiency upon IR irradiation, and the degree of secondary fragmentation may be controlled by adjusting the irradiation time. The resulting IRMPD mass spectra display a characteristic primary fragmentation pathway resulting from cleavage from only the nonreducing ends, thereby simplifying the determination of oligosaccharide sequence. As a result, the method should be generally applicable to the sequencing of even larger oligosaccharides.

\section{Acknowledgments}

The authors gratefully acknowledge funding from the Robert A. Welch Foundation (F1155) and the National Science Foundation (CHE-0315337 and CHE-0718320).

\section{References}

1. Varki, A. Biological Roles of Oligosaccharides: All of the Theories are Correct. Glycobiology 1993, 3, 97-130.

2. Linhardt, R. J.; Toida, T. Role of Glycosaminoglycans in Cellular Communication. Acc. Chem. Res. 2004, 37, 431-438.
3. Fannon, M.; Forsten, K. E.; Nugent, M. A. A Model for Regulation of Cellular Response. Biochemistry 2000, 39, 1434-1445.

4. Wu, Z. L.; Zhang, L.; Yabe, T.; Kuberan, B.; Beeler, D. L.; Love, A. Rosenberg, R. D. The Involvement of Heparan Sulfate (HS) in FGF1/ HS/FGFR1 Signaling Complex. J. Biol. Chem. 2003, 278, 17121-17129.

5. Dwek, R. A. Glycobiology: Toward Understanding the Function of Sugars. Chem. Rev. 1996, 96, 683-720.

6. Kukuruzinska, M. A.; Lennon, K. Protein N-Glycosylation: Molecular Genetics and Functional Significance. Crit. Rev. Oral. Biol. Med. 1998, 9, 415-448.

7. Helenus, A.; Aebi, M. Intracellular Functions of N-Linked Glycans. Science 2001, 291, 2364-2369.

8. Varki, A.; Cummings, R.; Esko, J.; Freeze, H.; Hart, G.; Marth, J. Essentials of Glycobiology, 1st ed.; Cold Spring Harbor Laboratory Press: Cold Spring Harbor, NY, 1999; p 653.

9. Gorelik, E.; Galili, U.; Raz, A. On the Role of Cell Surface Carbohydrates and Their Binding Proteins (Lectins) in Tumor Metastasis. Cancer Metast. Rev. 2001, 20, 245-277.

10. Dennis, J. W.; Granovsky, M.; Warren, C. E.Glycoprotein Glycosylation and Cancer Progression. Biochim. Biophys. Acta 1999, 1473, 21-34.

11. Rebbaa, A.; Chou, P. M.; Vucic, I.; Mirkin, B. L.; Tomita, T.; Bremer, E. G. Expression of Bisecting GlcNAc in Pediatric Brain Tumors and Its Association with Tumor Cell Response to Vinblastine. Clin. Cancer Res. 1999, 5, 3661-3668

12. Suzuki, Y.; Suzuki, M.; Ito, E.; Ishii, H.; Miseki, K.; Suzuki, A. Convenient and Rapid Analysis of Linkage Isomers of Fucose-Containing Oligosaccharides by Matrix-Assisted Laser Desorption/Ionization Quadrupole Ion Trap Time-of-Flight Mass Spectrometry. Glycoconj. J. 2005, 22, 427-431.

13. Yamagaki, T.; Ishizuka, Y.; Kawabata, S.; Nakanishi, H. Analysis of Glycosidic Linkages in Saccharide Compounds by Post-Source Decay Fragment Methods in Matrix-Assisted Laser Desorption/Ionization Time-of-Flight Mass Spectroscopy. Rapid Commun. Mass Spectrom. 1997, 11, 527-531.

14. Harvey, D. J. Matrix-Assisted Laser Desorption/Ionization Mass Spectrometry of Carbohydrates. Mass Spectrom. Rev. 1999, 18, 349-451.

15. Zaia, J. Mass Spectrometry of Oligosaccharides. Mass Spectrom. Rev. 2004, 23, 161-227.

16. Lancaster, K. S.; An, H. J.; Li, B.; Lebrilla, C. B. Interrogation of N-Linked Oligosaccharides Using Infrared Multiphoton Dissociation in FT-ICR Mass Spectrometry. Anal. Chem. 2006, 78, 4990-4997.

17. Penn, S. G.; Cancilla, M. T.; Lebrilla, C. B. Collision-Induced Dissociation of Branched Oligosaccharide Ions with Analysis and Calculation of Relative Dissociation Thresholds. Anal. Chem. 1996, 68, 2331-2339.

18. Park, Y.; Lebrilla, C. B. Application of Fourier Transform Ion Cyclotron Resonance Mass Spectrometry to Oligosaccharides. Mass Spectrom. Rev. 2005, 24, 232-264.

19. Pfenninger, A.; Karas, M.; Finke, B.; Stahl, B. Structural Analysis of Underivatized Neutral Human Milk Oligosaccharides in the Negative Ion Mode by Nano-Electrospray MS ${ }^{n}$, Part 1: Methodology. I. Am. Soc. Mass Spectrom. 2002, 13, 1331-1340.

20. Pfenninger, A.; Karas, M.; Finke, B.; Stahl, B. Structural Analysis of Underivatized Neutral Human Milk Oligosaccharides in the Negative Ion Mode by Nano-Electrospray MS ${ }^{n}$, Part 2: Application to Isomeric Mixtures. J. Am. Soc. Mass Spectrom. 2002, 13, 1341-1348.

21. Chai, W.; Piskarev, V.; Lawson, A. M. Negative-Ion Electrospray Mass Spectrometry of Neutral Underivatized Oligosaccharides. Anal. Chem. 2001, 73, 651-657.

22. Weiskopf, A. S.; Vouros, P.; Harvey, D. J. Electrospray Ionization-Ion Trap Mass Spectrometry for Structural Analysis of Complex N-Linked Glycoprotein Oligosaccharides. Anal. Chem. 1998, 70, 4441-4447.

23. Konig, S.; Leary, J. A. Evidence for Linkage Position Determination in Cobalt Coordinated Pentasaccharides Using Ion Trap Mass Spectrometry. J. Am. Soc. Mass Spectrom. 1998, 9, 1125-1134. 
24. Leavell, M. D.; Leary, J. A. Stabilization and Linkage Analysis of Metal-Ligated Sialic Acid Containing Oligosaccharides. J. Am. Soc. Mass Spectrom. 2001, 12, 528-536.

25. Ashline, D. J.; Lapadula, A. J.; Liu, Y.-H.; Lin, M.; Grace, M.; Pramanik, B.; Reinhold, V. N. Carbohydrate Structural Isomers Analyzed by Sequential Mass Spectrometry. Anal. Chem. 2007, 79, 3830-3842.

26. Asam, M. R.; Glish, G. L. Tandem Mass Spectrometry of Alkali Cationized Polysaccharides in a Quadrupole Ion Trap. J. Am. Soc. Mass Spectrom. 1997, 8, 987-995.

27. Asam, M. R.; Ray, K. L.; Glish, G. L. Collision-Induced Signal Enhancement: A Method To Increase Product Ion Intensities in MS/MS and MS Experiments. Anal. Chem. 1998, 70, 1831-1837.

28. Adamson, J. T.; Hakansson, K. Electron Capture Dissociation of Oligosaccharides Ionized with Alkali, Alkaline Earth, and Transition Metals. Anal. Chem. 2007, 79, 2901-2910

29. Wolff, J. J.; Amster, I. J.; Chi, L.; Linhardt, R. J. Electron Detachment Dissociation of Glycosaminoglycan Tetrasaccharides. J. Am. Soc. Mass Spectrom. 2007, 18, 234-244.

30. Devakumar, A.; Mechref, Y.; Kang, P.; Novotny, M. V.; Reilly, J. P. Laser-Induced Photofragmentation of Neutral and Acidic Glycans Inside an Ion-Trap Mass Spectrometer. Rapid Commun. Mass Spectrom. 2007, 21, 1452-1460.

31. Goldberg, D.; Bern, M.; Li, B. S.; Lebrilla, C. B. Automatic Determination of O-Glycan Structure from Fragmentation Spectra. J. Proteome Res. 2006, 5, 1429-1434.

32. Fukui, K.; Takada, Y.; Sumiyoshi, T.; Imai, T.; Takahashi, K. Infrared Multiphoton Dissociation Spectroscopic Analysis of Peptides and Oligosaccharides by Using Fourier Transform Ion Cyclotron Resonance Mass Spectrometry wit a Mid-Infrared Free-Electron Laser. J. Phys. Chem. B 2006, 110, 16111-16116.

33. Zhang, J.; Schubothe, K.; Li, B.; Russell, S.; Lebrilla, C. B. Infrared Multiphoton Dissociation of O-Linked Mucin-Type Oligosaccharides. Anal. Chem. 2005, 77, 208-214.

34. Shi, S.; Hendrickson, C. L.; Marshall, A. G.; Siegel, M. M.; Kong, F.; Carter, G. T. Structural Validation of Saccharomicins by High Resolution and High Mass Accuracy Fourier Transform-Ion Cyclotron ResonanceMass Spectrometry and Infrared Multiphoton Dissociation Tandem Mass Spectrometry. J. Am. Soc. Mass Spectrom. 1999, 10, 1285-1290.

35. Xie, Y.; Lebrilla, C. B. Infrared Multiphoton Dissociation of Alkali Metal-Coordinated Oligosaccharides. Anal. Chem. 2003, 75, 1590-1598.

36. Pikulski, M.; Wilson, J. J.; Aguilar, A.; Brodbelt, J. S. Amplification of Infrared Multiphoton Dissociation Efficiency in a Quadrupole Ion Trap Using IR-Active Ligands. Anal. Chem. 2006, 78, 8512-8517.

37. Wilson, J. J.; Brodbelt, J. S. Infrared Multiphoton Dissociation for Enhanced de Novo Sequence Interpretation of N-Terminal Sulfonated Peptides in a Quadrupole Ion Trap. Anal. Chem. 2006, 78, 6855-6862.

38. Crowe, M. C.; Brodbelt, J. S.; Goolsby, B. J.; Hergenrother, P. Characterization of Erythromycin Analogs by Collisionally Activated Dissociation and Infrared Multiphoton Dissociation in a Quadrupole Ion Trap. J. Am. Soc. Mass Spectrom. 2002, 13, 630-649.

39. Crowe, M. C.; Brodbelt, J. S. Infrared Multiphoton Dissociation (IRMPD) and Collisionally Activated Dissociation of Peptides in a Quadrupole Ion Trap with Selective IRMPD of Phosphopeptides. J. Am. Soc. Mass Spectrom. 2004, 15, 1581-1592.

40. Crowe, M. C.; Brodbelt, J. S. Differentiation of Phosphorylated and UnphosphorylatedPeptidesbyHigh-PerformanceLiquidChromatographyElectrospray Ionization-Infrared Multiphoton Dissociation in a Quadrupole Ion Trap. Anal. Chem. 2005, 77, 5726-5734.

41. Goolsby, B. J.; Brodbelt, J. S. Characterization of $\beta$-Lactams by Photodissociation and Collision-Activated Dissociation in a Quadrupole Ion Trap. J. Mass Spectrom. 1998, 33, 705-712.

42. Goolsby, B. J.; Brodbelt, J. S. Analysis of Protonated and Alkali Metal Cationized Aminoglycoside Antibiotics by Collision-Activated Dissociation and Infrared Multiphoton Dissociation in the Quadrupole Ion Trap. J. Mass Spectrom. 2000, 35, 1011-1024.
43. Goolsby, B. J.; Brodbelt, J. S. Tandem Infrared Multiphoton Dissociation and Collisionally Activated Dissociation Techniques in a Quadrupole Ion Trap. Anal. Chem. 2001, 73, 1270-1276.

44. Keller, K. M.; Brodbelt, J. S. Collisionally Activated Dissociation and Infrared Multiphoton Dissociation of Oligonucleotides in a Quadrupole Ion Trap. Anal. Biochem. 2004, 326, 200-210.

45. Vartanian, V. H.; Goolsby, B.; Brodbelt, I. S. Identification of Tetracycline Antibiotics by Electrospray Ionization in a Quadrupole Ion Trap. J. Am. Soc. Mass Spectrom. 1998, 9, 1089-1098.

46. Shen, J.; Brodbelt, J. S. Characterization of Ionophore-Metal Complexes by Infrared Multiphoton Photodissociation and Collision-Activated Dissociation in a Quadrupole Ion Trap Mass Spectrometer. Analyst 2000, $125,641-650$

47. Racine, A. H.; Payne, A. H.; Remes, P. M.; Glish, G. L. Thermally Assisted Collision-Induced Dissociation in a Quadrupole Ion Trap Mass Spectrometer. Anal. Chem. 2006, 78, 4609-4614.

48. Payne, A. H.; Glish, G. L. Thermally Assisted Infrared MPultiphoton photodissociation in a Quadrupole Ion Trap. Anal. Chem. 2001, 73, 3542-3548.

49. Hashimoto, Y.; Hasegawa, H.; Yoshinari, K.; Waki, I. Collision-Activated Infrared Multiphoton Dissociation in a Quadrupole Ion Trap Mass Spectrometer. Anal. Chem. 2003, 75, 420-425.

50. Boue, S. M.; Stephenson, J. L.; Yost, R. A. Pulsed Helium Introduction into a Quadrupole Ion Trap for Reduced Collisional Quenching During Infrared Multiphoton Dissociation of Electrosprayed ions. Rapid Commun. Mass Spectrom. 2000, 14, 1391-1397.

51. Hashimoto, Y.; Hasegawa, H.; Waki, L. High Sensitivity and Broad Dynamic Range Infrared Multiphoton Dissociation for a Quadrupole Ion Trap. Rapid Commun. Mass Spectrom. 2004, 18, 2255-2259.

52. Morelle, W.; Faid, V.; Michalski, J.-C. Structural Analysis of Permethylated Oligosaccharides Using Electrospray Ionization Quadrupole Time-of-Flight Tandem Mass Spectrometry and Deutero-Reduction. Rapid Commun. Mass Spectrom. 2004, 18, 2451-2464.

53. Viseux, N.; de Hoffmann, E.; Domon, B. Structural Assignment of Permethylated Oligosaccharide Subunits Using Sequential Tandem Mass Spectrometry. Anal. Chem. 1998, 70, 4951-4959.

54. Harvey, D. J. Electrospray Mass Spectrometry and Fragmentation of N-Linked Carbohydrates Derivatized at the Reducing Terminus. J. Am. Soc. Mass Spectrom. 2000, 11, 900-915.

55. Cheng, H.-L.; Pai, P.-J.; Her, G.-R. Linkage and Branch Determination of N-Linked Oligosaccharides Using Sequential Degradation/Closed-Ring Chromophore Labeling/Negative Ion Trap Mass Spectrometry. J. Am. Soc. Mass Spectrom. 2007, 18, 248-259.

56. Young, M. K.; Williams, D. Analysis of Neutral Isomeric Low Molecular Weight Carbohydrates Using Ferrocenyl Boronate Derivatization and Tandem Electrospray Mass Spectrometry. Rapid Commun. Mass Spectrom. 2000, 14, 2083-2091.

57. Yang, H.-J.; Chen, Y.-Z. Reaction Mass Spectrometry of Saccharides Using Areneboronic Acids as Reagents. J. Carbohydr. Chem. 1993, 12, 39-48.

58. Chen, H.; Cotte-Rodriguez, I.; Cooks, R. G. Cis-Diol Functional Group Recognition by Reactive Desorption Electrospray Ionization. Chem. Commun. 2006, 597-599.

59. Wong, A. W.; Cancilla, M. T.; Voss, L. R.; Lebrilla, C. B. Anion Dopant for Oligosaccharides in Matrix-Assisted Laser Desorption/Ionization Mass Spectrometry. Anal. Chem. 1999, 71, 205-211.

60. Zhu, L.; Shabbir, S. H.; Gray, M.; Lynch, V.; Sorey, S.; Anslyn, E. V. A Structural Investigation of the N-B Interaction in an o-(N,N-Dialkylaminomethyl) Arylboronate System. J. Am. Chem. Soc. 2006, 128, 1222-1232.

61. Zhu, L.; Anslyn, E. V. Facile Quantification of Enantiomeric Excess and Concentration with Indicator-Displacement Assays: An Example in the Analyses of $\alpha$-Hydroxyacids. J. Am. Chem. Soc. 2004, 126, 3676-3677.

62. Domon, B.; Costello, C. E. A Systematic Nomenclature for Carbohydrate Fragmentations in FAB-MS/MS Spectra of Glycoconjugates. Glycoconj. J. 1988, 5, 397-409. 